\title{
Optomechanics of Soft Materials
}

\section{Citation}

Bai, Ruobing, and Zhigang Suo. 2015. “Optomechanics of Soft Materials." J. Appl. Mech 82 (7) (June 3): 071011. doi:10.1115/1.4030324.

\section{Published Version}

doi:10.1115/1.4030324

\section{Permanent link}

http://nrs.harvard.edu/urn-3:HUL.InstRepos:17202192

\section{Terms of Use}

This article was downloaded from Harvard University's DASH repository, and is made available under the terms and conditions applicable to Open Access Policy Articles, as set forth at http:// nrs.harvard.edu/urn-3:HUL.InstRepos:dash.current.terms-of-use\#OAP

\section{Share Your Story}

The Harvard community has made this article openly available.

Please share how this access benefits you. Submit a story.

Accessibility 


\title{
Needleman Symposium: Optomechanics of Soft Materials
}

\section{Ruobing Bai}

School of Engineering and Applied Sciences, Kavli Institute for Bionano Science and Technology, Harvard University, Cambridge, Massachusetts 02138, USA

\section{Zhigang Suo'}

School of Engineering and Applied Sciences, Kavli Institute for Bionano Science and Technology, Harvard University, Cambridge, Massachusetts 02138, USA e-mail: suo@seas.harvard.edu ASME Fellow

\begin{abstract}
Some molecules change shape upon receiving photons of certain frequencies, but here we study light-induced deformation in ordinary dielectrics with no special optical effects. All dielectrics deform in response to light of all frequencies. We derive a dimensionless number to estimate when light can induce large deformation. For a structure made of soft dielectrics, with feature size comparable to the wavelength of light, the structure shapes the light, and the light deforms the structure. We study this two-way interaction between light and structure by combining the electrodynamics of light and the nonlinear mechanics of elasticity. We show that optical forces vary nonlinearly with deformation and readily cause optomechanical snapthrough instability. These theoretical ideas may help to create optomechanical devices of soft materials, complex shapes, and small features.
\end{abstract}

Keywords: optical forces, Maxwell stress, soft materials, optomechanics, optomechanical instability

\footnotetext{
${ }^{1}$ Corresponding author.
} 


\section{Introduction}

In 1862 Maxwell predicted that light of any frequency exerts a force on an illuminated surface [1]. The experimental confirmation came in 1900 [2], but such optical forces are feeble, and their practical uses have only arrived in recent decades, after lasers became widely available. Lasers are now used to move solid particles $[3,4]$, deform liquid interfaces $[5,6]$, bend slender rods $[7,8]$, and stretch cells $[9,10]$ (Fig. 1). Optomechanic devices have emerged as a vibrant field of research and development [11-15].

This paper develops the optomechanics of soft materials. Optical forces are feeble, but are stronger than forces that are feebler. We derive a dimensionless number to estimate when optical forces are large enough to induce large deformation in soft materials (Section 2). We then formulate a general method to calculate the light-induced large deformation (Section 3). Our task is simplified by two considerations: the optical properties of soft materials are insensitive to deformation, and mechanical motion is much slower than optical oscillation. Consequently, we calculate each increment of deformation using the Maxwell stress averaged over a single period of optical oscillation. On the other hand, because the feature size of a device may be comparable to the wavelength of the light, in general we do not average the Maxwell stress over space. We apply the method to a sheet of soft dielectric between two opposing lasers (Sections 4-7). We show that optical forces vary nonlinearly with deformation and readily cause optomechanical snap-through instability.

Light-induced deformation has also been studied in materials containing molecules capable of changing shape upon receiving photons of certain frequencies [16-21]. Such lightsensitive molecules achieve significant deformation at relatively low intensity of light. Here we focus on dielectrics with no special optical effects. Ordinary dielectrics achieve large lightinduced deformation only when the dielectrics are soft and the light is intense. Soft dielectrics constitute a large family of materials. This diversity will enable the choice of materials to meet various demands in applications, such as large deformation, facile fabrication, low cost, and 
biocompatibility. Maxwell stress has been used to calculate optical forces on stiff dielectrics [7, 8], and estimate deformation of soft dielectrics [22-24]. Optical forces enable two-way interactions between light and structures. For a structure made of soft dielectrics, the structure shapes the light, and the light deforms the structure. Here we formulate the optomechanics of soft materials that accounts for the two-way coupling between light and structure. We combine electrodynamics of light and nonlinear mechanics of elasticity.

Optical forces have been studied intensely, but have not been used to create devices of soft dielectrics. The potential, however, is enormous. Research in soft materials in the recent decade has led to a broad perspective: the softness enables materials to deform in response to stimuli, and the deformation provides functions [25]. Deformation links many stimuli to many functions. Thus, the deformation of a dielectric elastomer links a voltage to a force [26], the deformation of a hydrogel links a change in acidity in a solution to a change in the focal length of a lens [27], the swelling of an elastomer seals an oil well for hydraulic fracture [28, 29], and the deformation of a gel and an elastomer enables ionic music and ionic skin [30, 31]. Soft materials can be fabricated with feature sizes as small as several micrometers [32-35] and moduli as low as $\sim 10 \mathrm{OPa}[35-37]$. It is conceivable that optical forces will enable devices of soft materials, complex shapes, and small features. We hope that theoretical ideas described here will aid the creation of this technology.

\section{Magnitude of Optical Forces}

Light is a time-dependent electromagnetic field. Maxwell used the electromagnetic field to calculate the optical forces in the vacuum. His result is applicable to a medium of constant permittivity and permeability. The electromagnetic field in a given medium without free charge or current satisfies the Maxwell equations: $\nabla \times \mathbf{e}+\partial \mathbf{b} / \partial t=0, \nabla \times \mathbf{h}-\partial \mathbf{d} / \partial t=0, \nabla \cdot \mathbf{d}=\mathbf{0}$ and $\nabla \cdot \mathbf{b}=\mathbf{o}$. The field further satisfies the constitutive equations $\mathbf{d}=\boldsymbol{g} \mathbf{e}$ and $\mathbf{b}=\mu \mathbf{h}$, where $\varepsilon$ is 
the permittivity and $\mu$ is the permeability of the medium. At an interface between two media, the field satisfies the boundary conditions: $\mathbf{n} \cdot\left(\mathbf{b}_{2}-\mathbf{b}_{1}\right)=\mathbf{O}, \mathbf{n} \cdot\left(\mathbf{d}_{2}-\mathbf{d}_{1}\right)=\mathbf{O}, \mathbf{n} \times\left(\mathbf{e}_{2}-\mathbf{e}_{1}\right)=\mathbf{O}$ and $\mathbf{n} \times\left(\mathbf{h}_{2}-\mathbf{h}_{1}\right)=\mathbf{O}$, where $\mathbf{n}$ is the unit vector normal to the interface, and the subscripts indicate the two media. Associated with the electromagnetic field in each medium is the Maxwell stress [38]:

$$
T_{i j}=\varepsilon\left(e_{i} e_{j}-\frac{1}{2} e_{m} e_{m} \delta_{i j}\right)+\mu\left(h_{i} h_{j}-\frac{1}{2} h_{m} h_{m} \delta_{i j}\right)
$$

The Maxwell stress is a second-rank tensor, and is a field in the medium.

The Maxwell stress can be used to calculate the electromagnetic forces acting on an object. For example, consider two plates of conductor separated by a narrow gap of vacuum (Fig. 2). The voltage applied between the two plates causes an electric field in the vacuum, in the direction normal to the plates. Equation (1) predicts three components of Maxwell stress. One component is tensile in the vertical direction, and the other two components are compressive in the horizontal directions. The tensile Maxwell stress in the vertical direction causes the two plates to attract each other. This attractive electrostatic force balances a pair of mechanical forces that pull the plates apart. The horizontal components of the Maxwell stress are also commonly used in electromechanical systems. For example, when a dielectric sheet is partially placed in between the two plates, the horizontal Maxwell stress draws the sheet into the area between the two plates [39].

Equation (1) indicates that Maxwell stress scales with $\varepsilon e^{2}$ and $\mu h^{2}$, as well as with the square root of their product, $\sqrt{\varepsilon \mu} e h$. Recall that $c=1 / \sqrt{\varepsilon \mu}$ is the speed of light in the medium, and $\mathbf{e} \times \mathbf{h}$ is the flux of energy (i.e., the energy of light crossing a plane normal to the direction of propagation per unit time per unit area). Consequently, the magnitude of the Maxwell stress scales as $P /\left(c a^{2}\right)$, where $P$ is the power and $a$ the width of a beam of light. For representative values of a laser, $P=0.1 \mathrm{~W}, a=1 \mu \mathrm{m}$ and $c=3 \times 10^{8} \mathrm{~m} / \mathrm{s}$, we estimate the Maxwell stress 
$P /\left(\mathrm{ca}^{2}\right) \sim 3 \mathrm{odPa}$. By comparison, the elastic modulus is $\sim 1 \mathrm{O}^{11} \mathrm{~Pa}$ for stiff materials such as silicon, but is $\sim 100$ Pa for a soft gel. Thus, the Maxwell stress due to a laser can cause small strains in stiff materials, but large strains in soft materials.

We compare the magnitudes of the optical forces to those of other forces by forming dimensionless numbers. For example, to stretch a cell by a large strain, the Maxwell stress $P /\left(\mathrm{ca}^{2}\right)$ needs to be comparable to the elastic modulus of the cell, $G$. This consideration leads to a dimensionless number $P /\left(c a^{2} G\right)$. Similar considerations lead to dimensionless numbers that compare the optical force with other types of forces, including forces due to thermal fluctuation, viscous flow, surface tension, elastic bending, and elastic stretching (Table 1). The larger the numbers are, the greater the effect of optical forces will be.

Estimates of these dimensionless numbers agree with existing experimental observations (Fig. 1). For example, optical forces can overcome elasticity and cause deformation in two ways: bend stiff materials and stretch soft materials. In bending a stiff material, such as a slender rod of silicon, choosing power $P=0.1 \mathrm{~W}$, width $a=31 \mathrm{~mm}$, length $L=30 / \mathrm{m}$ and modulus $G \approx 8 \propto G P a$, we get $(L / a)^{4} P /\left(c a^{2} G\right) \approx 4$. In stretching a soft material such as a cell, choosing power $P=0.7 \mathrm{~W}$, size $a=1$ qu $\mathrm{m}$ and modulus $G \approx 1 \mathrm{OPa}$, we get $P /\left(c a^{2} G\right) \approx 2$. Both estimates show that optical forces are sufficient to cause deformation, agreeing with experimental observations $[7,9]$. The two dimensionless numbers differ by the factor $(L / a)^{4}$, where $L$ is the length and $a$ the width of the beam. This factor is huge for a representative value of $L / a \sim 1$ oo, enabling the optical forces to bend a stiff material, even though the modulus $G$ is large. The high moduli of stiff materials, however, limit substantial change of shape to a single type: bending of slender rods. By contrast, soft materials can achieve large deformation of many types, and may potentially lead to devices of many kinds. The remainder of this paper focuses on the optomechanics of soft materials. 


\section{Optomechanics of Two-Way, Light-Structure Interaction}

We now develop the optomechanics of soft materials to account for the two-way, lightstructure interaction. Our task is simplified by two considerations. First, the permittivity and permeability of a soft dielectric are insensitive to deformation [40]. A soft dielectric is a three dimensional network of polymer. The number of crosslinks between the polymer chains is much smaller than the number of monomers constituting the chains. The crosslinks turn a liquid into a solid, but negligibly affect permittivity and permeability. That is, the electromagnetic property of a crosslinked dielectric is essentially the same as that of the corresponding liquid dielectric. Here we assume that the permittivity and permeability are independent of deformation. This assumption allows us to calculate the optical forces in soft materials using the Maxwell stress.

Second, optical frequency is much larger than mechanical frequency. The frequency of mechanical vibration scales as $a^{-1} \sqrt{G / \rho}$, where $a$ is the feature size, $G$ the elastic modulus, and $\rho$ the density. For representative values, $a=10^{-6} \mathrm{~m}, G=10^{3} \mathrm{~Pa}$ and $\rho=10^{3} \mathrm{~kg} / \mathrm{m}^{3}$, the frequency of mechanical vibration is $\sim 10^{6} \mathrm{~Hz}$, which is much lower than the frequency of light, $\sim 1 \mathrm{O}^{14} \mathrm{~Hz}$. That is, each period of mechanical oscillation corresponds to a large number of periods of electromagnetic oscillation. Consequently, each state of deformation is determined by the Maxwell stress averaged over a single period of the electromagnetic oscillation. On the other hand, because the feature size of a device may be comparable to the wavelength of the light, in general we do not average the Maxwell stress over space.

Under these simplifications, we develop the optomechanics of soft materials by combining the nonlinear mechanics of elasticity and the electrodynamics of light. The nonlinear mechanics of elasticity is well-established [41]. A material body is represented by a sum of many small pieces. Each piece is named after its place $\mathbf{X}$ when the body is in the undeformed state. The piece $\mathbf{X}$ occupies a place $\mathbf{x}$ when the body is in a deformed state at time $t$. The function $\mathbf{x}=\mathbf{x}(\mathbf{X}, t)$ describes the history of the deformation of the body. The deformation gradient is 
$F_{i K}=\partial x_{i}(\mathbf{X}, t) / \partial X_{K}$. The nominal stress $s_{i k}(\mathbf{X}, t)$ relates to the true stress $\sigma_{i j}(\mathbf{x}, t)$ as $\sigma_{i j}=s_{i k} F_{j k} / \operatorname{det} \mathbf{F}$. On a piece of volume $\mathrm{d} V(\mathbf{X})$, we prescribe mass $\rho(\mathbf{X}) \mathrm{d} V$ and force $\widetilde{\mathbf{B}}(\mathbf{X}, t) \mathrm{d} V$. On a piece of area $\mathrm{d} A(\mathbf{X})$ normal to the unit vector $N_{K}$, we prescribe a force $\widetilde{T}_{i} \mathrm{~d} A(\mathbf{X})$. We add " " to distinguish the body force from the magnetic field, and the traction from the Maxwell stress. The balance of forces requires that $\partial s_{i K} / \partial X_{K}+\widetilde{B}_{i}=\rho \partial^{2} x_{i} / \partial t^{2}$ in the volume and that $s_{i k} N_{K}=\widetilde{T}_{i}$ on the surface.

The electrodynamics of light is also well-established [42]. In a deformed state of the body, the electric field $\mathbf{e}(\mathbf{x}, t)$ is sinusoidal in time, $\mathbf{e}(\mathbf{x}, t)=\operatorname{Re}[\mathbf{E}(\mathbf{x}) \exp (i \omega t)]$, where $\omega$ is the frequency of the light, and complex-valued field $\mathbf{E}(\mathbf{x})$ is the phasor of the electric field. Phasors for the other fields are similarly defined, $\mathbf{H}(\mathbf{x}), \mathbf{D}(\mathbf{x})$ and $\mathbf{B}(\mathbf{x})$. We assume that the material is dielectric, with no free charge or current density. Under this assumption, the Maxwell equations in terms of phasors are $\nabla \cdot \mathbf{D}=0, \nabla \times \mathbf{E}=-i \omega \mathbf{B}, \nabla \cdot \mathbf{B}=\mathbf{0}$, and $\nabla \times \mathbf{H}=i \omega \mathbf{D}$. The Maxwell stress affects deformation through its average over a period of electromagnetic oscillation, $\left\langle T_{i j}\right\rangle=(\omega / 2 \pi) \int_{0}^{2 \pi / \omega} T_{i j} \mathrm{~d} t$. An evaluation of this integral gives that [43]

$$
\left\langle T_{i j}\right\rangle=\frac{1}{2} \operatorname{Re}\left[\varepsilon\left(E_{i} E_{j}^{*}-\frac{1}{2} E_{m} E_{m}^{*} \delta_{i j}\right)+\mu\left(H_{i} H_{j}^{*}-\frac{1}{2} H_{m} H_{m}^{*} \delta_{i j}\right)\right]
$$

where $E_{i}^{*}$ and $H_{i}^{*}$ are the complex-conjugates of $E_{i}$ and $H_{i}$, respectively.

We write the true stress $\sigma_{i j}$ as the sum of that due to the elastic deformation and that due to the time-averaged Maxwell stress:

$$
\sigma_{i j}=\frac{F_{j K}}{\operatorname{det} \mathbf{F}} \frac{\partial W(\mathbf{F})}{\partial F_{i K}}+\left\langle T_{i j}\right\rangle
$$

where $W(\mathbf{F})$ is the Helmholtz free energy associated with the stretching of the material. For an incompressible material, $\operatorname{det} \mathbf{F}=\mathbf{1}$ and (3) becomes that 


$$
\sigma_{i j}=F_{j K} \frac{\partial W(\mathbf{F})}{\partial F_{i K}}-\Pi \delta_{i j}+\left\langle T_{i j}\right\rangle
$$

where $\Pi$ is the Lagrange multiplier, to be determined as a part of the solutions to boundaryvalue problems.

To account for the two-way interaction between the light and the structure, such a boundary-value problem is solved in incremental steps. Starting with an undeformed state, we apply mechanical and optical loads of small amplitudes, determine the optical field by solving an eigenvalue problem over the body of a fixed configuration, and determine the increment of the displacement field by solving a boundary-value problem. Then we use the increment of displacement to update the configuration of the body, and increase the loads by a small amount. We repeat the procedure for many steps to obtain the final deformed state. The optical field is Eulerian, and the elastic field is Lagrangian. This mixed specification poses an interesting challenge for general numerical method, which we do not pursue in this paper. Instead, we focus on a special case to describe optomechancial phenomena of potential significance in applications.

\section{A Sheet of Soft Dielectric between Two Opposing Lasers}

We apply the theory to a thin sheet of a dielectric between two opposing lasers (Fig. 3). The refractive index is $n$ in the sheet, and is $n^{\text {out }}$ in the outside medium. The sheet has the dimensions $\left(L_{1}, L_{2}, L_{3}\right)$ in the undeformed state, and dimensions $\left(l_{1}, l_{2}, l_{3}\right)$ in the deformed state. The effect of surface tension is taken to be negligible.

The two lasers have the same frequency and intensity, propagating as plane waves in the opposite directions. Let $\left(E_{L}, H_{L}\right)$ be the field of the laser from the left side of the dielectric, and $\left(E_{R}, H_{R}\right)$ be the field of the laser from the right side of the dielectric. We further assume that the fields $E_{L}$ and $E_{R}$ are symmetric with respect to the mid-plane of the dielectric. Consequently, 
the two lasers generate optical forces of equal magnitude in opposite directions, and keep the mid-plane of the sheet stationary in space. Let the coordinates $\left(x_{1}, x_{2}, x_{3}\right)$ coincide with the directions of the fields and propagations, with $x_{3}=0$ at the mid-plane of the dielectric. The fields of the lasers are $E_{L}=E_{\mathrm{o}} \exp \left(i k^{\text {out }} x_{3}\right), H_{L}=\sqrt{\varepsilon^{\text {out }} / \mu} E_{\mathrm{o}} \exp \left(i k^{\text {out }} x_{3}\right), E_{R}=E_{\mathrm{o}} \exp \left(-i k^{\text {out }} x_{3}\right)$ and $H_{R}=\sqrt{\varepsilon^{\text {out }} / \mu} E_{\mathrm{o}} \exp \left(-i k^{\text {out }} x_{3}\right)$, where $\varepsilon^{\text {out }}=\left(n^{\text {out }}\right)^{2} \varepsilon_{\mathrm{o}}$ is the permittivity of the outside medium, $\mu$ is the permeability of both the dielectric and the outside medium, $E_{\mathrm{o}}$ is the amplitude of the electric field, $k^{\text {out }}=n^{\text {out }} \omega / c_{\mathrm{o}}$ is the wavenumber of the lasers, $\omega$ is the frequency of the lasers, and $c_{0}$ is the speed of light in the vacuum.

The thickness of the dielectric is comparable to the wavelength of the lasers in the sheet, $\Lambda=2 \pi c_{\mathrm{o}} /(n \omega)$, but the in-plane dimensions of the sheet are much larger than $\Lambda$. Thus, we will consider only homogeneous deformation of principal stretches $\lambda_{1}=l_{1} / L_{1}, \lambda_{2}=l_{2} / L_{2}$ and $\lambda_{3}=l_{3} / L_{3}$

Both in the dielectric and outside, the components of the electric field are $E_{1}=E$ and $E_{2}=E_{3}=\mathrm{O}$, and the components of the magnetic field are $H_{2}=H$ and $H_{1}=H_{3}=\mathrm{O}$. Equation (2) shows that only the principal Maxwell stresses are nonzero:

$$
\left\langle T_{1}\right\rangle=-\left\langle T_{2}\right\rangle=\frac{1}{4}\left(\varepsilon|E|^{2}-\mu|H|^{2}\right),\left\langle T_{3}\right\rangle=-\frac{1}{4}\left(\varepsilon|E|^{2}+\mu|H|^{2}\right)
$$

The dielectric is assumed to be incompressible, $\lambda_{1} \lambda_{2} \lambda_{3}=1$, so that (4) becomes that

$$
\begin{gathered}
\sigma_{1}-\sigma_{3}=\lambda_{1} \frac{\partial W\left(\lambda_{1}, \lambda_{2}\right)}{\partial \lambda_{1}}+\left\langle T_{1}\right\rangle-\left\langle T_{3}\right\rangle \\
\sigma_{2}-\sigma_{3}=\lambda_{2} \frac{\partial W\left(\lambda_{1}, \lambda_{2}\right)}{\partial \lambda_{2}}+\left\langle T_{2}\right\rangle-\left\langle T_{3}\right\rangle
\end{gathered}
$$

We represent the elasticity of the dielectric using the neo-Hookean model [44]:

$$
W\left(\lambda_{1}, \lambda_{2}\right)=\frac{G}{2}\left(\lambda_{1}^{2}+\lambda_{2}^{2}+\left(\lambda_{1} \lambda_{2}\right)^{-2}-3\right)
$$


where $G$ is the shear modulus of the dielectric.

For the time being, we assume that the sheet is subject to no external mechanical forces. The balance of forces in the $x_{1}$ and $x_{2}$ directions requires that the resultant forces vanish, $\int_{0}^{l_{3}} \sigma_{1} d x_{3}=\int_{0}^{l_{3}} \sigma_{2} d x_{3}=0$. The balance of force in the $x_{3}$ direction requires that the stress in the dielectric equals the Maxwell stress, $\sigma_{3}=\left\langle T_{3}^{\text {out }}\right\rangle$. Inserting these boundary conditions to (6) and (7), and using (8), we get

$$
\begin{aligned}
& t_{1}-t_{3}=G\left(\lambda_{1}^{2}-\lambda_{3}^{2}\right) \\
& t_{2}-t_{3}=G\left(\lambda_{2}^{2}-\lambda_{3}^{2}\right)
\end{aligned}
$$

where

$$
t_{1}=-\frac{1}{l_{3}} \int_{0}^{l_{3}}\left\langle T_{1}\right\rangle \mathrm{d} x_{3}, t_{2}=-t_{1}, t_{3}=\left\langle T_{3}^{\text {out }}\right\rangle-\left\langle T_{3}\right\rangle
$$

Equation (11) writes the optical forces as the equivalent mechanical stresses acting on the dielectric (Fig. 3c). Once the optical field is known in the dielectric and the outside medium, (5) gives the Maxwell stresses, and (9)-(11) determine the stretches.

\section{Total Reflection}

Consider the special case that the two lasers are fully reflected at the surfaces of the sheet. This total reflection may be achieved by coating the sheet with conducting films. In this case, no optical field exists inside the sheet, and only the Maxwell stress in the outside medium, $\left\langle T_{3}^{\text {out }}\right\rangle$, causes the sheet to deform. The electric field on the right hand side of the sheet is the superposition of the incident wave and the reflected wave,

$$
\begin{gathered}
E_{\text {right }}=E_{\mathrm{o}}\left[\exp \left(-i k^{\text {out }} x_{3}\right)-\exp \left(i k^{\text {out }}\left(x_{3}+l_{3}\right)\right)\right] \\
H_{\text {right }}=\sqrt{\varepsilon^{\text {out }} / \mu} E_{\mathrm{o}}\left[\exp \left(-i k^{\text {out }} x_{3}\right)+\exp \left(i k^{\text {out }}\left(x_{3}+l_{3}\right)\right)\right]
\end{gathered}
$$

Consequently, (2) gives $\left\langle T_{3}^{\text {out }}\right\rangle=-\varepsilon^{\text {out }} E_{\mathrm{o}}^{2}$, known as the radiation pressure. The same radiation 
pressure exists on the left-hand side. The radiation pressure is independent of the thickness of the dielectric and the polarization of the lasers. These conclusions are consistent with existing experiments and calculations $[45,46]$.

\section{Sheet Optically Matched with the Medium Outside}

Next consider the case that the sheet and the outside medium are optically matched, $n=n^{\text {out }}$. If we only apply the laser on the right side, $\left(E_{R}, H_{R}\right)$, Equation (2) gives $\left\langle T_{1}\right\rangle=\left\langle T_{2}\right\rangle=\mathrm{O}$ and $\left\langle T_{3}\right\rangle=\left\langle T_{3}^{\text {out }}\right\rangle=-\varepsilon E_{0}^{2} / 2$, so that the net optical forces vanish. However, if we apply two opposing lasers, the optical field will be the superposition of the two lasers, giving $E=2 E_{\mathrm{o}} \cos \left(k x_{3}\right)$ and $H=-2 i \sqrt{\varepsilon / \mu} E_{\mathrm{o}} \sin \left(k x_{3}\right)$. Equations (2) and (11) give the equivalent stresses $t_{1}=-\varepsilon E_{\mathrm{o}}^{2}\left(\sin k l_{3}\right) /\left(k l_{3}\right), t_{2}=-t_{1}$ and $t_{3}=0$. As the thickness of the sheet changes, $t_{1}$ changes between tensile and compressive, and vanishes as $k l_{3} \rightarrow \infty$. Applying the equivalent stresses to (9) and (10), we obtain the state of deformation (Fig. 4). When the thickness of the sheet is the multiples of the half-wavelength of the lasers, the optical forces vanish. To maintain the same deformation, the amplitude of the lasers needs to be infinitely large.

The optical force varies nonlinearly with the deformation, and can readily cause optomechanical instability. Such instability has been observed in optomechanical systems involving slender rods of stiff materials [15], but has not been reported in optomechanical systems of soft materials. To illustrate the basic behavior, consider a sheet placed between the two opposing lasers, clamped at the top and the bottom, and then pulled by a mechanical force $f$ (Fig. 5a). The clamps constrain the sheet in the horizontal direction, $\lambda_{1}=1$, and the sheet is incompressible, $\lambda_{3}=1 / \lambda_{2}$. Thus, (7) gives

$$
\varepsilon E_{\mathrm{o}}^{2} \frac{\sin k l_{3}}{k l_{3}}+\frac{f}{l_{1} l_{3}}=G\left(\lambda_{2}^{2}-\frac{1}{\lambda_{2}^{2}}\right)
$$


We plot the force-stretch relation for a sheet between lasers of a fixed amplitude (Fig. 5b). As the stretch $\lambda_{2}$ increases, the thickness of the dielectric $l_{3}$ decreases, and the optical force decreases to negative, returns to positive, and then decreases again. Consequently, the force-stretch curve reaches the peak, goes down, and then goes up again. This curve corresponds to a snap-through instability. When the force $f$ is programed to increase gradually, the sheet initially elongates gradually. Upon reaching the peak of the curve, the sheet suddenly stretches greatly. Furthermore, when the force is programed to increase and then decrease gradually, the sheet snaps forward and backward, undergoing hysteresis. We plot Fig. 5b using a sheet of the undeformed thickness $L_{3}=2.0 \Lambda$. If we use a thicker sheet, the force-stretch curve will have multiple peaks and valleys, since the deformed thickness will go through several periods of the sinusoidal optical force. We have also tried various other types of mechanical constraints and loads, and found that the snap-through instability occurs in many configurations.

\section{Sheet Optically Mismatched with the Medium Outside}

Now consider the case that the sheet and the outside medium are optically mismatched, $n \neq n^{\text {out }}$. Each incident wave will cause both transmitted and reflected waves. The net optical field can be solved analytically, giving [42, 47]

$$
\begin{aligned}
& E_{\text {in }}=\frac{2(1+\rho) \cos \left(k x_{3}\right)}{\left(1-\rho^{2} e^{-2 i k l_{3}}\right)} E_{\mathrm{o}}\left[e^{i\left(k^{\text {out }}-k\right)_{3} / 2}-\rho e^{-2 k l_{3} l_{3}} e^{i\left(k^{\text {out }}+k\right) l_{3} / 2}\right] \\
& H_{\text {in }}=\sqrt{\frac{\varepsilon}{\mu}} \frac{2 i(1+\rho) \sin \left(k x_{3}\right)}{\left(1-\rho^{2} e^{-2 k k l_{3}}\right)} E_{\mathrm{o}}\left[e^{i\left(k^{\mathrm{out}}-k\right) l_{3} / 2}-\rho e^{-2 i k l_{3}} e^{i\left(k^{\text {out }}+k\right) l_{3} / 2}\right] \\
& E_{\text {right }}=E_{\mathrm{o}} e^{-i k^{\text {out } x_{3}}}+\frac{\left(\rho+e^{-i k l_{3}}\right)\left(1-\rho e^{-i k l_{3}}\right)}{\left(1-\rho^{2} e^{-2 i k l_{3}}\right)} E_{\mathrm{o}} e^{i k^{\text {out }}\left(x_{3}+l_{3}\right)} \\
& H_{\text {right }}=\sqrt{\frac{\varepsilon^{\text {out }}}{\mu}}\left[E_{0} e^{-k k^{\text {out }} x_{3}}-\frac{\left(\rho+e^{-i k l_{3}}\right)\left(1-\rho e^{-i k l_{3}}\right)}{\left(1-\rho^{2} e^{-2 i k l_{3}}\right)} E_{\mathrm{o}} e^{i k^{\text {out }}\left(x_{3}+l_{3}\right)}\right]
\end{aligned}
$$

where $\rho=\left(n^{\text {out }}-n\right) /\left(n^{\text {out }}+n\right)$ measures the optical mismatch and $k=n \omega / c_{\mathrm{o}}$ is the wavenumber 
of the light inside the sheet. Here $E_{\text {in }}$ and $H_{\text {in }}$ are the net field inside the sheet, and $E_{\text {right }}$ and $H_{\text {right }}$ are the net field on the right side of the sheet. Because of the symmetry of the setup, we do not list $E_{\text {left }}$ and $H_{\text {left }}$.

Inserting the optical field into (5), we obtain the Maxwell stresses:

$$
\begin{gathered}
\left\langle T_{1}\right\rangle_{\text {in }}=\varepsilon E_{\mathrm{o}}^{2} \frac{(1+\rho)^{2}\left[1+\rho^{2}-2 \rho \cos \left(k l_{3}\right)\right]}{1+\rho^{4}-2 \rho^{2} \cos \left(2 k l_{3}\right)} \cos \left(2 k x_{3}\right) \\
\left\langle T_{2}\right\rangle_{\text {in }}=-\left\langle T_{1}\right\rangle_{\text {in }},\left\langle T_{3}\right\rangle_{\text {in }}=-\varepsilon E_{\mathrm{o}}^{2} \frac{(1+\rho)^{2}\left[1+\rho^{2}-2 \rho \cos \left(k l_{3}\right)\right]}{1+\rho^{4}-2 \rho^{2} \cos \left(2 k l_{3}\right)} \\
\left\langle T_{1}\right\rangle_{\text {right }}=\varepsilon^{\text {out }} E_{\mathrm{o}}^{2} \operatorname{Re}\left\{\frac{\left(\rho+e^{\text {ikl }}\right)\left(1-\rho e^{i k l_{3}}\right)}{\left(1-\rho^{2} e^{2 i k l_{3}}\right)} e^{-2 k^{\text {out } l_{3}} e^{-2 i k^{\text {out }} x_{3}}}\right\} \\
\left\langle T_{2}\right\rangle_{\text {right }}=-\left\langle T_{1}\right\rangle_{\text {right }},\left\langle T_{3}\right\rangle_{\text {right }}=-\varepsilon^{\text {out }} E_{\mathrm{o}}^{2}
\end{gathered}
$$

where $\varepsilon=n^{2} \varepsilon_{\mathrm{o}}$ is the permittivity of the dielectric. Because of the symmetry of the setup, we do not list the Maxwell stress on the left side of the sheet. Note that the through-thickness Maxwell stress, $\left\langle T_{3}\right\rangle$, is constant in each medium, independent of the positions. In general, the values of $\left\langle T_{3}\right\rangle$ in different media are different. This difference causes a nonzero equivalent stress in the through-thickness direction. The in-plane Maxwell stresses are sinusoidal functions of $x_{3}$. We plot the Maxwell stresses when the sheet is at two specific thicknesses, $l_{3}=\Lambda$ and $l_{3}=1.5 \Lambda$ (Fig. 6). When $l_{3}=\Lambda$, the value of $\left\langle T_{3}\right\rangle$ in the sheet differs from that outside. This difference gives a nonzero equivalent stress, even though the in-plane Maxwell stresses average out. By contrast, when $l_{3}=1.5 \Lambda$, the value of $\left\langle T_{3}\right\rangle$ in the sheet is the same as that outside, so that the equivalent stress vanishes.

The equivalent stresses are

$$
t_{1}=-\varepsilon E_{\mathrm{o}}^{2} \frac{(1+\rho)^{2}\left[1+\rho^{2}-2 \rho \cos \left(k l_{3}\right)\right]}{1+\rho^{4}-2 \rho^{2} \cos \left(2 k l_{3}\right)} \frac{\sin \left(k l_{3}\right)}{k l_{3}}, t_{2}=-t_{1}
$$




$$
t_{3}=\varepsilon E_{\mathrm{o}}^{2} \frac{(1+\rho)^{2}\left[1+\rho^{2}-2 \rho \cos \left(k l_{3}\right)\right]}{1+\rho^{4}-2 \rho^{2} \cos \left(2 k l_{3}\right)}-\varepsilon^{\mathrm{out}} E_{\mathrm{o}}^{2}
$$

Inserting the optical forces into (9) and (10), we determine the stretches of the sheet. We note several differences between the optically mismatched case (Fig. 7) and the optically matched case (Fig. 6). As $l_{3} \rightarrow \Lambda$ the optical mismatch ensures that the lasers cause an optical force, so that $\varepsilon_{\mathrm{o}} E_{\mathrm{o}}^{2}$ now is finite. For a thick dielectric, the in-plane optical forces vanish, but the optical mismatch causes a finite through-thickness optical force in general. The optical force pulls the sheet in the thickness direction when $n>n^{\text {out }}$, but compresses the sheet in the thickness direction when $n<n^{\text {out }}$.

\section{Maxwell Stress as a Boundary Condition}

We have added the Maxwell stress as the part of the stress-stretch relation in (3). We have also transformed the Maxwell stress into the equivalent stress in the calculation of the dielectric sheet. This method is generally applicable. For a dielectric of constant permittivity and permeability, with no free charge or current, the Maxwell equations ensure that $\nabla \cdot \mathbf{T}=c^{-2} \partial(\mathbf{e} \times \mathbf{h}) / \partial t[42,46]$. If we further assume that the Maxwell stress affects deformation through its time average, and the electromagnetic field is a sinusoidal function of $t$, we find that $\langle\partial(\mathbf{e} \times \mathbf{h}) / \partial t\rangle=\mathbf{O}$ and $\nabla \cdot\langle\mathbf{T}\rangle=\mathbf{0}$. We let $\sigma_{i j}^{\mathrm{e}}=F_{j K} \partial W(\mathbf{F}) / \partial F_{i K}-\Pi \delta_{i j}$ be the stress due to elasticity, leave the equation $F_{i K}=\partial x_{i}(\mathbf{X}, t) / \partial X_{K}$ unchanged, and change the equation for the balance of forces to $\partial s_{i K}^{\mathrm{e}} / \partial X_{K}+\widetilde{B}_{i}=\rho \partial^{2} x_{i} / \partial t^{2}$, where the nominal stress $s_{i K}^{\mathrm{e}}$ satisfies $\sigma_{\ddot{j}}^{\mathrm{e}}=F_{j K} s_{i K}^{\mathrm{e}} / \operatorname{det} \mathbf{F}$. These equations are the same as the equations in nonlinear elasticity. However, the boundary condition now should be modified to be $\sigma_{i j}^{\mathrm{e}} n_{j}=\tilde{t}_{i}+\left(\left\langle T_{\ddot{i}}^{\text {out }}\right\rangle-\left\langle T_{i j}\right\rangle\right) n_{j}$. In addition to the mechanical traction $\tilde{t}_{i}$, the difference of the Maxwell stress in the medium outside and that in the dielectric should be added on the boundary. We can solve such a problem by deriving the electromagnetic 
field in deformed state, calculating the Maxwell stress field, prescribing the difference of the Maxwell stress on the boundary, and applying the governing equations of nonlinear elasticity.

\section{Concluding Remarks}

Our calculations show that optical forces can cause large deformation of soft materials. We show that optical forces vary nonlinearly with deformation and readily cause optomechancial snap-through instability. We describe the optomechanics of light-induced large deformation in structures of any shape. Optomechanics combines electrodynamics of light and nonlinear mechanics of elasticity. For a soft dielectric with no special optical effects, its optomechanical behavior is fully specified by three material constants: the permittivity, permeability, and shear modulus. Structures made of soft dielectrics are sensitive to geometry, creating an enormous space for innovation in conceptual design, computation, and fabrication.

Optical forces enable two-way, light-structure interaction. A structure shapes a light, and the light deforms the structure. One potential application of the optomechanics of soft materials concerns using light to control light. For example, in the field of structural colors, a structure of a feature size comparable to optical wavelength interacts with light through scattering, diffraction or interference [48-55]. One may use soft materials to make the structures, and then use optical forces to deform the structures and change the optical behavior. One can also conceive optical circuits reconfigurable by optical forces. A second potential application concerns the transduction between light and other stimuli. Many soft materials deform in response to stimuli such as stress [53-55], electric field [26, 51], acidity and humidity [27-29, 52]. Thus, the deformation of soft materials enables the transduction between stimuli of different types. It is conceivable, for example, that devices can be created using ordinary soft dielectrics to perform optochemistry. We hope that the theoretical ideas developed here will aid in the creation of devices using light-induced large deformation. 


\section{Acknowledgments}

This work is supported by MRSEC (DMR-0820484). This paper is prepared for the special issue that celebrates the $7 \mathrm{O}^{\text {th }}$ birthday of Professor Alan Needleman.

\section{References}

[1] Maxwell, J. C., 1862, "III. On Physical Lines of Force," Philosophical Magazine Series 4, 23(151), pp. 12-24. DOI: 10.1080/14786446208643207

[2] Lebedew, P., 1901, "Untersuchungen Über Die Druckkräfte Des Lichtes," Annalen der Physik, 311(11), pp. 433-458. DOI: 10.1002/andp.19013111102

[3] Ashkin, A., Dziedzic, J. M., Bjorkholm, J. E., and Chu, S., 1986, "Observation of a SingleBeam Gradient Force Optical Trap for Dielectric Particles," Optics Letters, 11(5), pp. 288-290. http://dx.doi.org/10.1364/OL.11.000288

[4] Chu, S., 1991, "Laser Manipulation of Atoms and Particles," Science, 253(5022), pp. 861-866. DOI: $10.1126 /$ science.253.5022.861

[5] Ashkin, A., and Dziedzic, J., 1973, "Radiation Pressure on a Free Liquid Surface," Physical Review Letters, 30(4), pp. 139-142. http://dx.doi.org/10.1103/PhysRevLett.30.139

[6] Casner, A., and Delville, J.-P., 2001, "Giant Deformations of a Liquid-Liquid Interface Induced by the Optical Radiation Pressure," Physical Review Letters, 87(5), pp. 054503. http://dx.doi.org/10.1103/PhysRevLett.87.054503

[7] Povinelli, M. L., Loncar, M., Ibanescu, M., Smythe, E. J., Johnson, S. G., Capasso, F., and Joannopoulos, J. D., 2005, "Evanescent-Wave Bonding between Optical Waveguides," Optics Letters, 30(22), pp. 3042-3044. http://dx.doi.org/10.1364/OL.30.003042

[8] Li, M., Pernice, W. H. P., Xiong, C., Baehr-Jones, T., Hochberg, M., and Tang, H. X., 2008, "Harnessing Optical Forces in Integrated Photonic Circuits," Nature, 456(7221), pp. 480-484. DOI: $10.1038 /$ nature07545 
[9] Guck, J., Ananthakrishnan, R., Moon, T., Cunningham, C., and Käs, J., 200o, "Optical Deformability of Soft Biological Dielectrics," Physical Review Letters, 84(23), pp. 5451-5454. http://dx.doi.org/10.1103/PhysRevLett.84.5451

[10] Dao, M., Lim, C. T., and Suresh, S., 2003, "Mechanics of the Human Red Blood Cell Deformed by Optical Tweezers," Journal of the Mechanics and Physics of Solids, 51(11-12), pp. 2259-2280. DOI: 10.1016/j.jmps.2003.09.019

[11] Neuman, K. C., and Block, S. M., 2004, "Optical Trapping," Review of Scientific Instruments, 75(9), pp. 2787-2809. http://dx.doi.org/10.1063/1.1785844

[12] Kippenberg, T. J., and Vahala, K. J., 2008, "Cavity Optomechanics: Back-Action at the Mesoscale," Science, 321(5893), pp. 1172-1176. DOI: 10.1126/science.1156032

[13] Van Thourhout, D., and Roels, J., 2010, "Optomechanical Device Actuation through the Optical Gradient Force," Nat Photon, 4(4), pp. 211-217. DOI: 10.1038/nphoton.2010.72

[14] Aspelmeyer, M., Meystre, P., and Schwab, K., 2012, "Quantum Optomechanics," Physics Today, 65(7), pp. 29-35. http://dx.doi.org/10.1063/PT.3.1640

[15] Ren, M., Huang, J., Cai, H., Tsai, J. M., Zhou, J., Liu, Z., Suo, Z., and Liu, A.-Q., 2013, "Nano-Optomechanical Actuator and Pull-Back Instability," ACS nano, 7(2), pp. 1676-1681. DOI: $10.1021 / \mathrm{nn} 3056687$

[16] Finkelmann, H., Nishikawa, E., Pereira, G., and Warner, M., 2001, "a New OptoMechanical Effect in Solids," Physical Review Letters, 87(1), pp. 015501. http://dx.doi.org/10.1103/PhysRevLett.87.015501

[17] Hogan, P., Tajbakhsh, A., and Terentjev, E., 2002, "Uv Manipulation of Order and Macroscopic Shape in Nematic Elastomers," Physical Review E, 65(4), pp. 041720. http://dx.doi.org/10.1103/PhysRevE.65.041720

[18] Yu, Y., Nakano, M., and Ikeda, T., 2003, "Photomechanics: Directed Bending of a Polymer Film by Light," Nature, 425(6954), pp. 145-145. DOI:10.1038/425145a 
[19] Warner, M., and Mahadevan, L., 2004, "Photoinduced Deformations of Beams, Plates, and

$\begin{array}{llllll}\text { Films," } & \text { Physical } & \text { Review } & \text { Letters, } & 92(13), & \text { pp. }\end{array}$

http://dx.doi.org/10.1103/PhysRevLett.92.134302

[20] Dunn, M. L., 2007, "Photomechanics of Mono-and Polydomain Liquid Crystal Elastomer Films," Journal of applied physics, 102(1), pp. 013506. http://dx.doi.org/10.1063/1.2745063

[21] Krishnan, D., and Johnson, H. T., 2014, "Light-Induced Deformation in a Liquid Crystal Elastomer Photonic Crystal," Journal of the Mechanics and Physics of Solids, 62(0), pp. 48-56. DOI:10.1016/j.jmps.2013.08.013

[22] Xu, F., Lock, J., Gouesbet, G., and Tropea, C., 2009, "Optical Stress on the Surface of a Particle: Homogeneous Sphere," Physical Review A, 79(5), pp. 053808. http://dx.doi.org/10.1103/PhysRevA.79.053808

[23] Boyde, L., Ekpenyong, A., Whyte, G., and Guck, J., 2012, "Comparison of Stresses on Homogeneous Spheroids in the Optical Stretcher Computed with Geometrical Optics and Generalized Lorenz-Mie Theory," Applied optics, 51(33), pp. 7934-7944. http://dx.doi.org/10.1364/AO.51.007934

[24] Yang, M., Ren, K. F., Wu, Y., and Sheng, X., 2014, "Computation of Stress on the Surface of a Soft Homogeneous Arbitrarily Shaped Particle," Physical Review E, 89(4), pp. 043310. http://dx.doi.org/10.1103/PhysRevE.89.043310

[25] Suo, Z., 2012, "Mechanics of Stretchable Electronics and Soft Machines," MRS Bulletin, 37(03), pp. 218-225. http://dx.doi.org/10.1557/mrs.2012.32

[26] Pelrine, R., Kornbluh, R., Pei, Q., and Joseph, J., 2000, "High-Speed Electrically Actuated Elastomers with Strain Greater Than 100\%," Science, 287(5454), pp. 836-839. DOI: 10.1126/science.287.5454.836 
[27] Zalachas, N., Cai, S., Suo, Z., and Lapusta, Y., 2013, "Crease in a Ring of a Ph-Sensitive Hydrogel Swelling under Constraint," International Journal of Solids and Structures, 5o(6), pp. 920-927. DOI:10.1016/j.ijsolstr.2012.11.015

[28] Cai, S., Lou, Y., Ganguly, P., Robisson, A., and Suo, Z., 2010, "Force Generated by a Swelling Elastomer Subject to Constraint," Journal of Applied Physics, 107(10), pp. 103535. http://dx.doi.org/10.1063/1.3428461

[29] Liu, Q., Wang, Z., Lou, Y., and Suo, Z., 2014, "Elastic Leak of a Seal," Extreme Mechanics Letters, In Press. DOI: 10.1016/j.eml.2014.10.001

[30] Keplinger, C., Sun, J.-Y., Foo, C. C., Rothemund, P., Whitesides, G. M., and Suo, Z., 2013, "Stretchable, Transparent, Ionic Conductors," Science, 341(6149), pp. 984-987. DOI: $10.1126 /$ science.1240228

[31] Sun, J.-Y., Keplinger, C., Whitesides, G. M., and Suo, Z., 2014, "Ionic Skin," Advanced Materials, 26(45), pp. 7608-7614. DOI: 10.1002/adma.201403441

[32] Zarzar, L. D., Kim, P., Kolle, M., Brinker, C. J., Aizenberg, J., and Kaehr, B., 2011, "Direct Writing and Actuation of Three-Dimensionally Patterned Hydrogel Pads on Micropillar Supports," Angewandte Chemie, 123(40), pp. 9528-9532. DOI: 10.1002/ange.201102975

[33] Nash, M. E., Carroll, W. M., Foley, P. J., Maguire, G., Connell, C. O., Gorelov, A. V., Beloshapkin, S., and Rochev, Y. A., 2012, "Ultra-Thin Spin Coated Crosslinkable Hydrogels for Use in Cell Sheet Recovery-Synthesis, Characterisation to Application," Soft Matter, 8(14), pp. 3889-3899. DOI: 10.1039/C2SMo6466A

[34] Wu, G., Xia, Y., and Yang, S., 2014, "Buckling, Symmetry Breaking, and Cavitation in Periodically Micro-Structured Hydrogel Membranes," Soft Matter, 10(9), pp. 1392-1399. DOI: 10.1039/C3SM51640G

[35] Cui, J., Björnmalm, M., Liang, K., Xu, C., Best, J. P., Zhang, X., and Caruso, F., 2014, "Super-Soft Hydrogel Particles with Tunable Elasticity in a Microfluidic Blood Capillary Model," Advanced Materials, 26(43), pp. 7295-7299. DOI: 10.1002/adma.201402753 
[36] Chakrabarti, A., and Chaudhury, M. K., 2013, "Direct Measurement of the Surface Tension of a Soft Elastic Hydrogel: Exploration of Elastocapillary Instability in Adhesion," Langmuir, 29(23), pp. 6926-6935. DOI: 10.1021/la401115j

[37] Style, R. W., Boltyanskiy, R., German, G. K., Hyland, C., Macminn, C. W., Mertz, A. F., Wilen, L. A., Xu, Y., and Dufresne, E. R., 2014, "Traction Force Microscopy in Physics and Biology," Soft Matter, 10(23), pp. 4047-4055. DOI: 10.1039/C4SMo0264D

[38] Maxwell, J. C., 1873, A Treatise on Electricity and Magnetism, Clarendon Press Series, Clarendon Press, Oxford.

[39] Feynman, R. P., Leighton, R. B. J. A., and Sands, M. L., 1963, The Feynman Lectures on Physics, Addison-Wesley Pub. Co., Reading, Mass. ISBN: 0201020106

[40] Zhao, X., Hong, W., and Suo, Z., 2007, "Electromechanical Hysteresis and Coexistent States in Dielectric Elastomers," Physical Review B, 76(13), pp. 134113. http://dx.doi.org/10.1103/PhysRevB.76.134113

[41] Truesdell, C. A and Noll, W. A., 2004, The Non-Linear Field Theories of Mechanics, Springer Berlin Heidelberg: Imprint: Springer, Berlin, Heidelberg. ISBN: 9783662103883 | 9783662103883 | 9783642057014

[42] Stratton, J. A., 1941, Electromagnetic Theory, International Series in Physics, McGrawHill book company, Inc., New York, London.

[43] Ploschner, M., Mazilu, M., Krauss, T. F., and Dholakia, K., 2010, "Optical Forces near a Nanoantenna," Journal of Nanophotonics, 4(1), pp. $\quad$ 041570-041570-13. http://dx.doi.org/10.1117/1.3332850

[44] Treloar, L. R. G., 1943, "The Elasticity of a Network of Long-Chain Molecules - II," Transactions of the Faraday Society, 39(o), pp. 241-246. DOI: 10.1039/TF9433900241

[45] Jones, R. V., and Richards, J. C. S., 1954, "The Pressure of Radiation in a Refracting Medium," Proceedings of the Royal Society of London A: Mathematical, Physical and Engineering Sciences, 221(1147), pp. 480-498. DOI: 10.1098/rspa.1954.0043 
[46] Novotny, L., and Hecht, B., 2006, Principles of Nano-Optics, Cambridge University Press, Cambridge; New York. ISBN: 0521832241

[47] Orfanidis, S. J., 2002, Electromagnetic Waves and Antennas, Last modified July 2, 2014, http://www.ece.rutgers.edu/ orfanidi/ewa/

[48] Biró, L. P., and Vigneron, J. P., 2011, "Photonic Nanoarchitectures in Butterflies and Beetles: Valuable Sources for Bioinspiration," Laser \& Photonics Reviews, 5(1), pp. 27-51. DOI: 10.1002/lpor.200900018

[49] Chung, K., Yu, S., Heo, C.-J., Shim, J. W., Yang, S.-M., Han, M. G., Lee, H.-S., Jin, Y., Lee, S. Y., Park, N., and Shin, J. H., 2012, "Flexible, Angle-Independent, Structural Color Reflectors Inspired by Morpho Butterfly Wings," Advanced Materials, 24(18), pp. 2375-2379. DOI: $10.1002 / \operatorname{adma.201200521~}$

[50] Raut, H. K., Ganesh, V. A., Nair, A. S., and Ramakrishna, S., 2011, "Anti-Reflective Coatings: A Critical, in-Depth Review," Energy \& Environmental Science, 4(10), pp. 3779-3804. DOI: 10.1039/C1EE01297E

[51] Aschwanden, M., and Stemmer, A., 2006, "Polymeric, Electrically Tunable Diffraction Grating Based on Artificial Muscles," Optics Letters, 31(17), pp. 2610-2612. http://dx.doi.org/10.1364/OL.31.002610

[52] Ye, G., and Wang, X., 2010, "Polymer Diffraction Gratings on Stimuli-Responsive Hydrogel Surfaces: Soft-Lithographic Fabrication and Optical Sensing Properties," Sensors and Actuators B: Chemical, 147(2), pp. 707-713. DOI:10.1016/j.snb.2010.03.052

[53] Wilbur, J. L., Jackman, R. J., Whitesides, G. M., Cheung, E. L., Lee, L. K., and Prentiss, M. G., 1996, "Elastomeric Optics," Chemistry of Materials, 8(7), pp. 1380-1385. DOI: 10.1021/cm950579d

[54] Hiroshi, F., 2011, "Tunable Structural Color in Organisms and Photonic Materials for Design of Bioinspired Materials," Science and Technology of Advanced Materials, 12(6), pp. 064704. DOI:10.1088/1468-6996/12/6/064704 
Journal of Applied Mechanics

[55] Haque, M. A., Kurokawa, T., Kamita, G., Yue, Y., and Gong, J. P., 2011, "Rapid and Reversible Tuning of Structural Color of a Hydrogel over the Entire Visible Spectrum by Mechanical Stimulation," Chemistry of Materials, 23(23), pp. 5200-5207. DOI: $10.1021 / \mathrm{cm} 2021142$ 


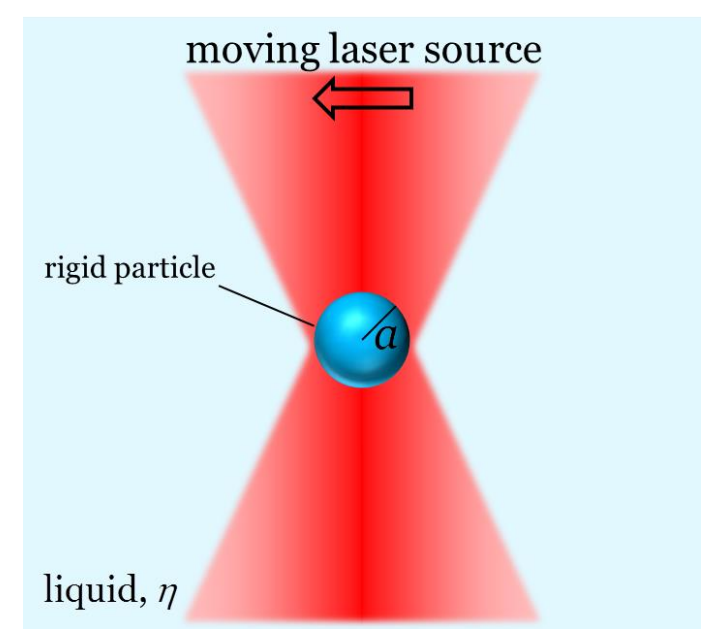

(a) Trap and move a rigid particle

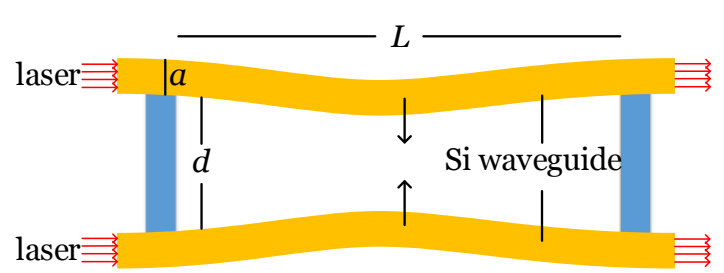

(c) Bend a stiff material

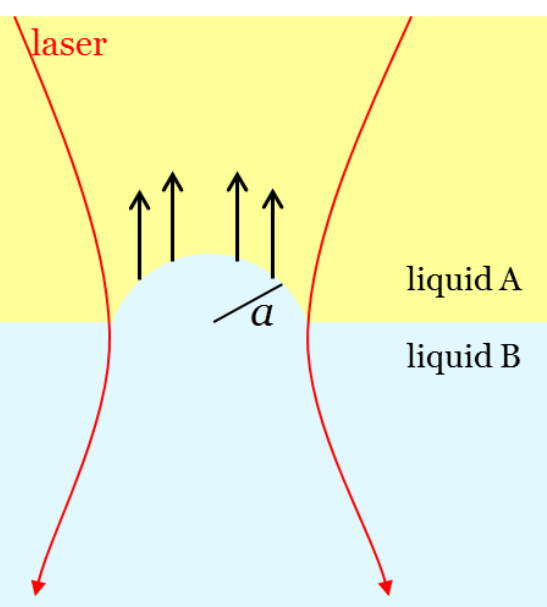

(b) Deform an interface between liquids

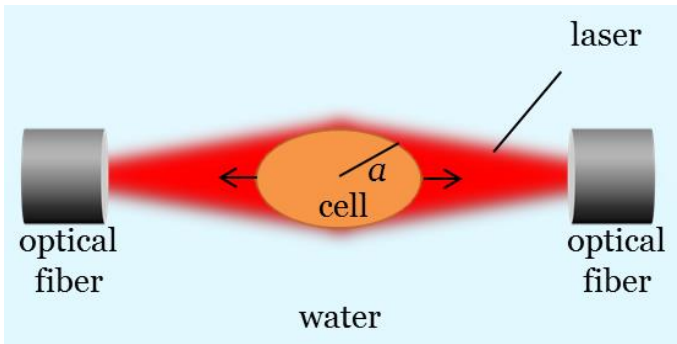

(d) Stretch a soft material

Fig. 1 Optical forces cause motions of various kinds. (a) Optical tweezers trap a rigid particle and move it in a liquid. (b) A beam of light deforms the interface between two liquids. (c) Light in two waveguides causes an evanescent optical field in the space between the waveguides, and bends them. (d) Two beams of laser stretch a cell. 


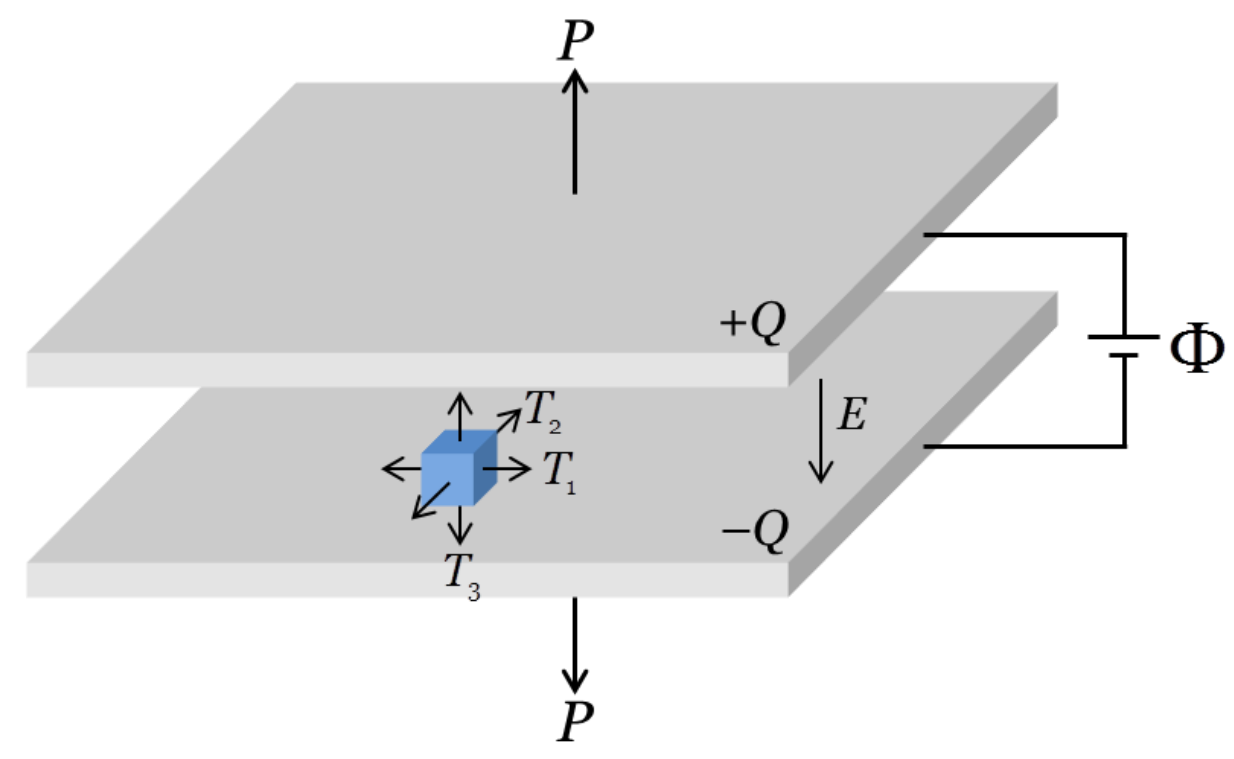

Fig. 2 The Maxwell stress in the vacuum between two parallel plates of conductor separated by a narrow gap of vacuum. The applied voltage $\Phi$ induces electric charges $\pm Q$ on the two plates, as well as an electric field $E$ and a Maxwell-stress field in the vacuum between the two plates. The Maxwell stress is a tensor with three principal components, tensile in the vertical direction, and compressive in the two horizontal directions. The three components have the same magnitude, $\varepsilon_{0} E^{2} / 2$, where $\varepsilon_{0}$ is the permittivity of the vacuum. The tensile Maxwell stress in the vertical direction causes the two plates to attract each other. This attractive electrostatic force is balanced by a mechanical force $P$. 


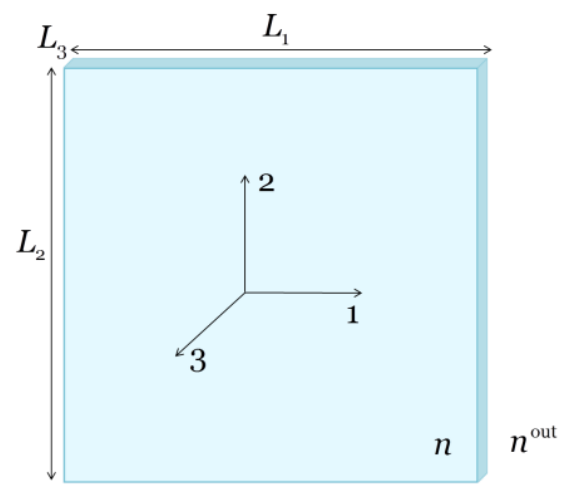

(a) undeformed state

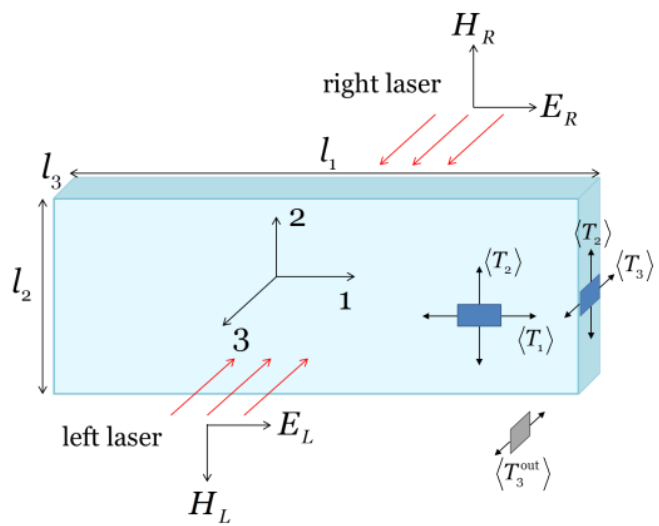

(b) deformed state

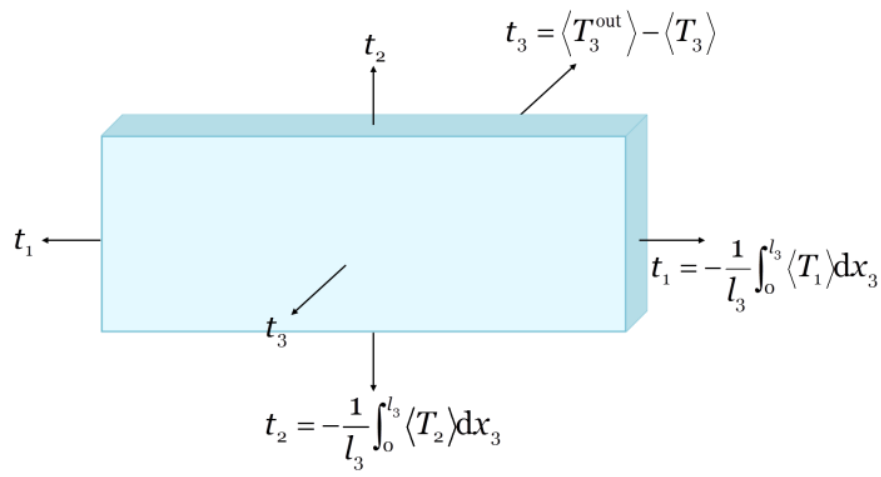

(c) equivalent mechanical stress

Fig. 3 Two anti-parallel lasers deform a thin sheet of a soft dielectric. The refractive index is $n$ in the dielectric, and $n^{\text {out }}$ in the outside medium. (a) In the undeformed state, the dielectric has the dimensions $\left(L_{1}, L_{2}, L_{3}\right)$. (b) In the deformed state, the dielectric deforms to the dimensions $\left(l_{1}, l_{2}, l_{3}\right)$. The electromagnetic fields of the left and right lasers are $\left(E_{L}, H_{L}\right)$ and $\left(E_{R}, H_{R}\right)$, respectively. The lasers generate the Maxwell stress in the dielectric and outside. (c) The Maxwell stress causes equivalent mechanical stress acting on the dielectric. 


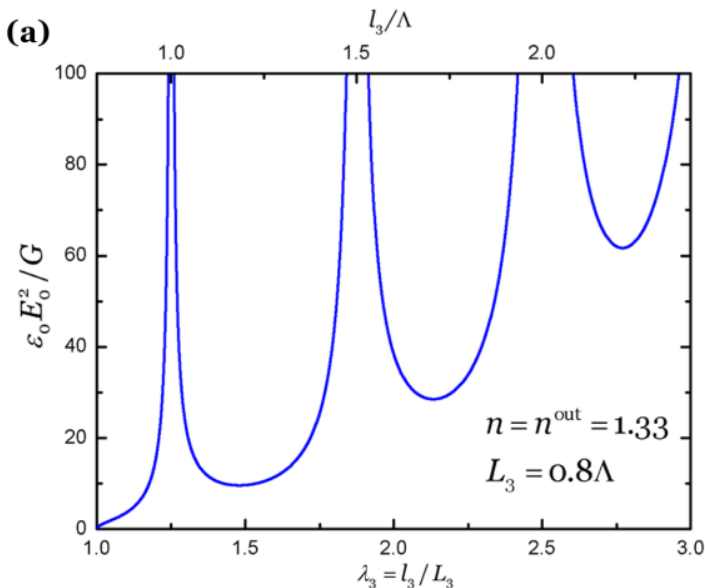

(c)

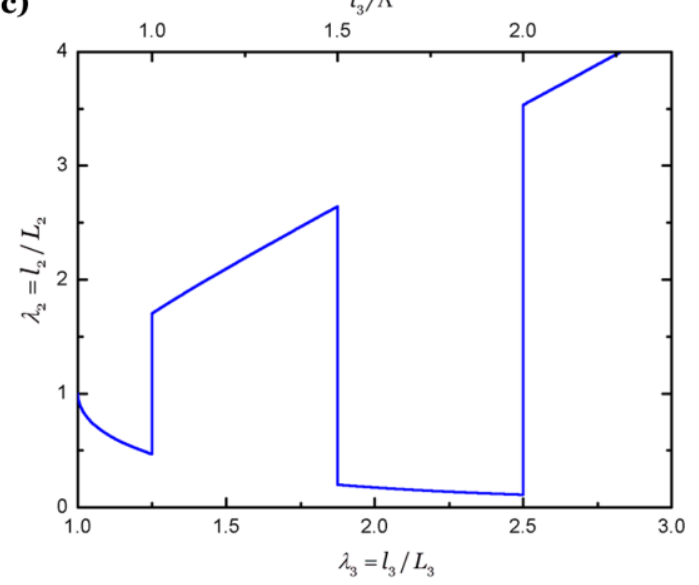

(b)

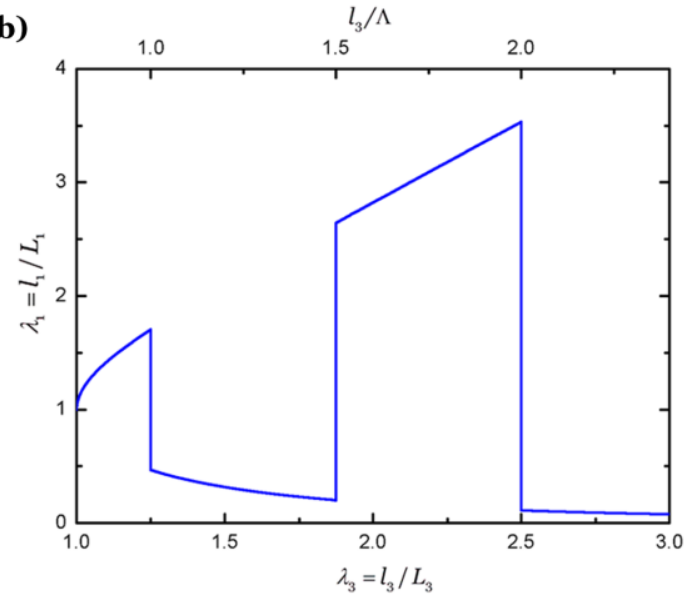

(d)

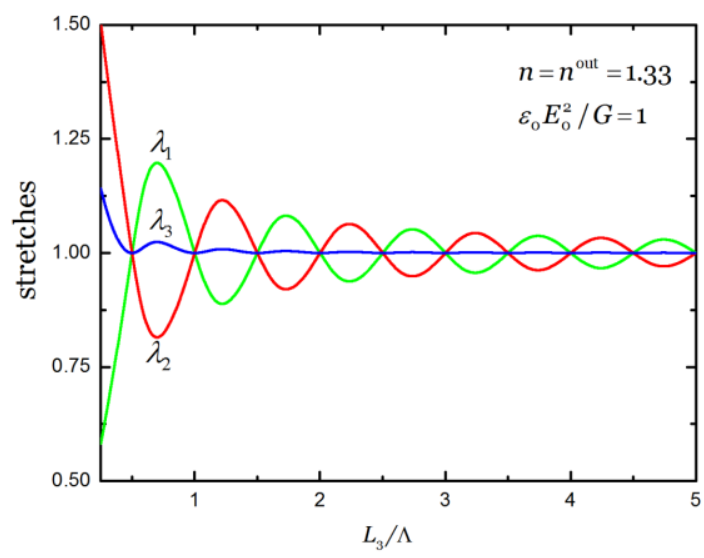

Fig. 4 Deformation induced by optical forces in a dielectric optically matched to the outside. $\Lambda$ is the wavelength of the laser inside the dielectric. (a) The out-of-plane stretch as a function of the amplitude of the input optical field. When the thickness of the deformed dielectric, $l_{3}$, approaches the multiples of the half wavelength, $0.5 \Lambda, 1.0 \Lambda, 1.5 \Lambda \ldots$, all components of the optical force approach zero, so that amplitude of the input optical field, $E_{\mathrm{o}}$, becomes larger and larger to maintain the deformation. (b) and (c) The in-plane stretches as functions of the out-of-plane stretch. (d) The stretches depend on the thickness of the undeformed dielectric, $L_{3}$. The dielectric is optically matched with the outside, so that the out-of-plane component of the optical force vanishes, $t_{3}=\left\langle T_{3}^{\text {out }}\right\rangle-\left\langle T_{3}\right\rangle=0$, but the in-plane components of the optical force in general do not vanish, $t_{1}=-\left(1 / l_{3}\right) \int_{0}^{l_{3}}\left\langle T_{1}\right\rangle \mathrm{d} x_{3}$ and $t_{2}=-\left(1 / l_{3}\right) \int_{0}^{l_{3}}\left\langle T_{2}\right\rangle \mathrm{d} x_{3}$. When $L_{3}=0.5 \Lambda, 1.0 \Lambda, 1.5 \Lambda$ and so on, all components of the optical force vanish, and the dielectric does not deform. When $L_{3} / \Lambda \rightarrow \infty$, the in-plane components of the optical force average out, $t_{1}=t_{2} \rightarrow 0$, and the dielectric does not deform. 
(a)

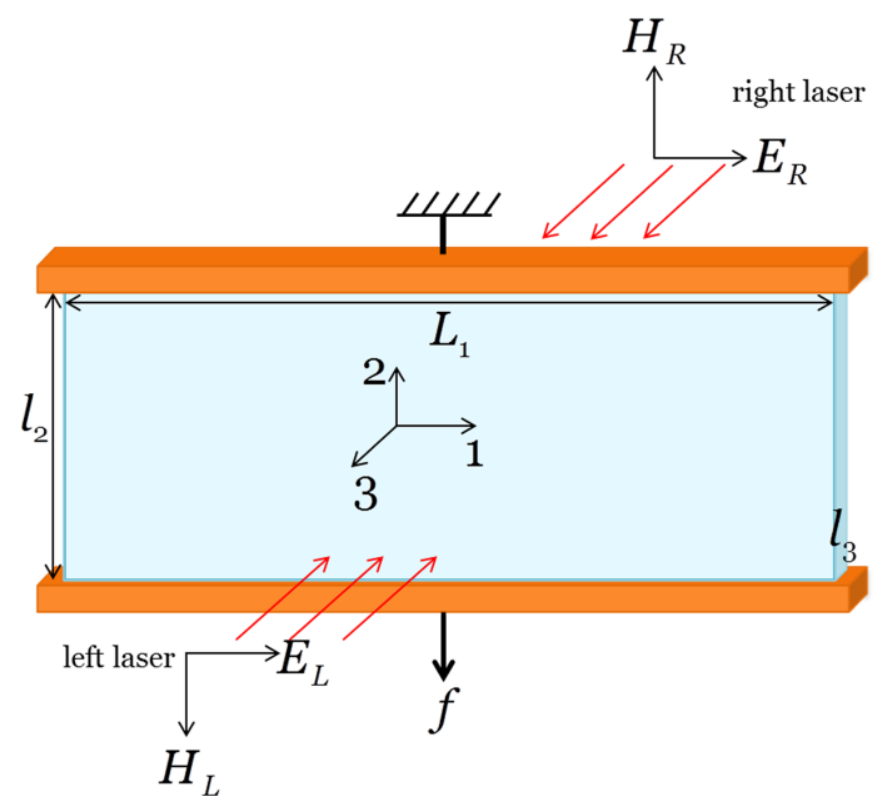

(b)

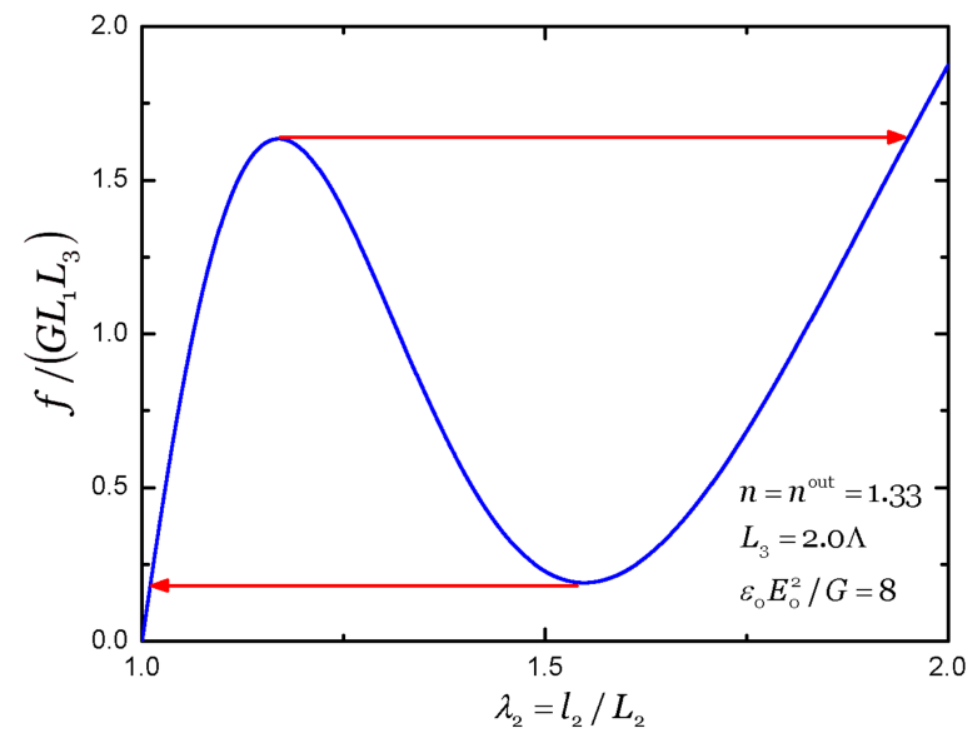

Fig. 5 Optomechanical snap-through instability. (a) A thin sheet of dielectric is placed in the optical field of two anti-parallel lasers. The dielectric is clamped at the top and bottom, and is pulled by a mechanical force $f$ in the vertical direction. (b) In the presence of the optical field, the force-displacement curve is not monotonic, leading to a snap-through instability. 
(a)

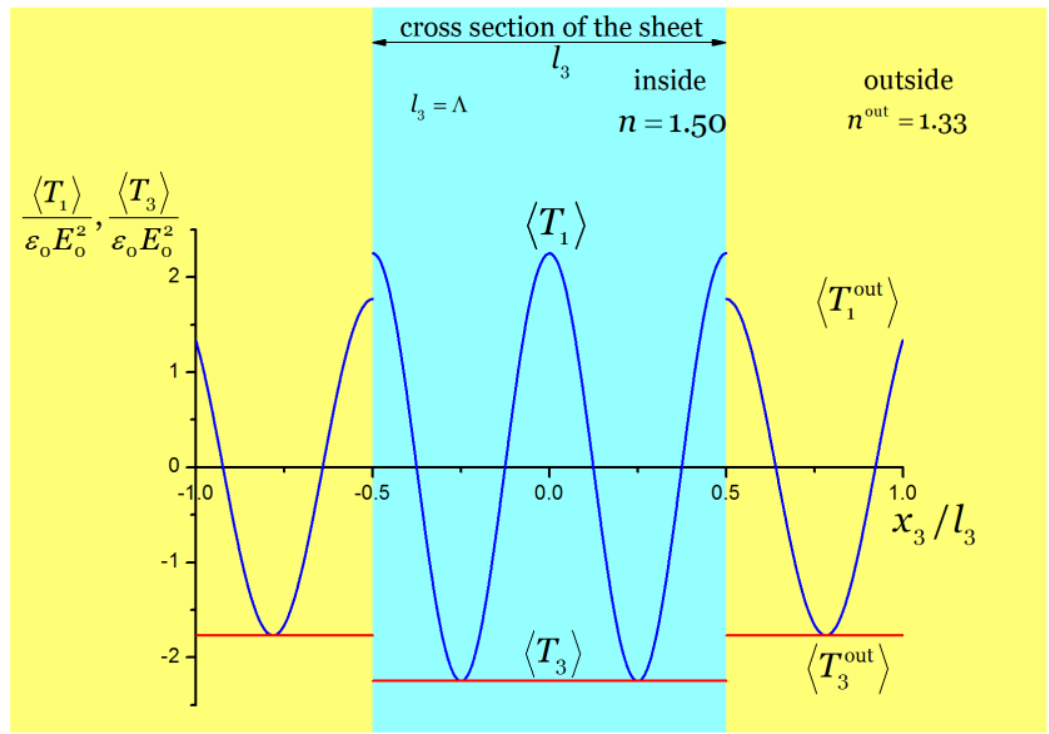

(b)

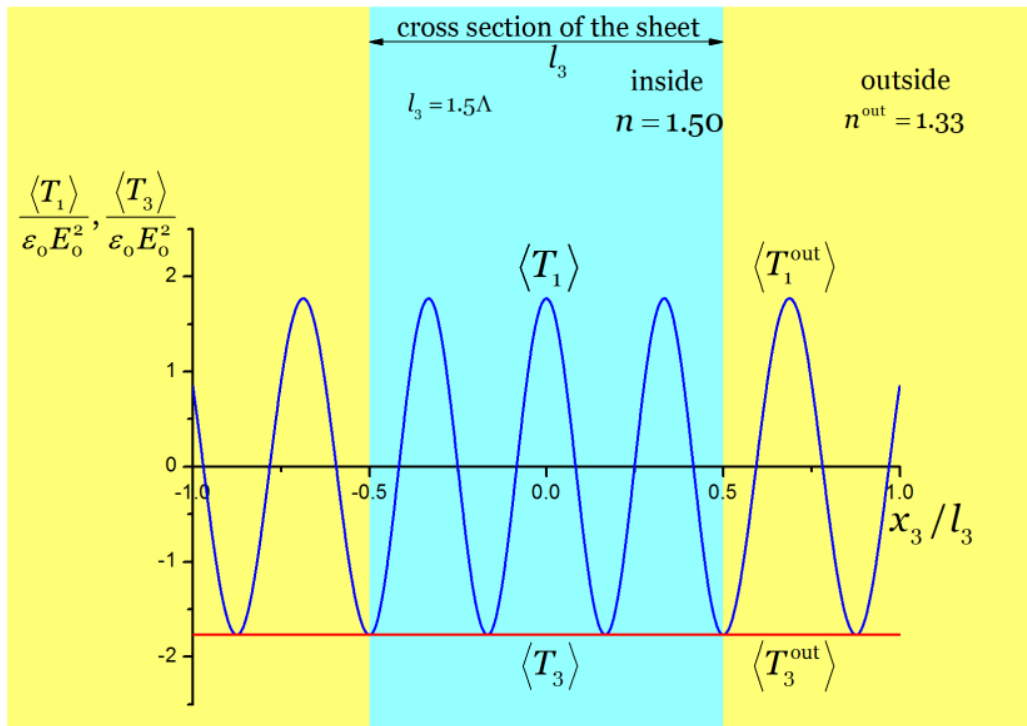

Fig. 6 Maxwell stresses in a dielectric optically mismatched with the outside. Because $\left\langle T_{2}\right\rangle=-\left\langle T_{1}\right\rangle$, only $\left\langle T_{1}\right\rangle$ and $\left\langle T_{3}\right\rangle$ are plotted. (a) When $l_{3}=\Lambda$, both $\left\langle T_{1}\right\rangle$ and $\left\langle T_{2}\right\rangle$ average to zero, but $\left\langle T_{3}^{\text {out }}\right\rangle \neq\left\langle T_{3}\right\rangle$. (b) When $l_{3}=1.5 \Lambda$, all three components of the optical force vanish. 
(a)

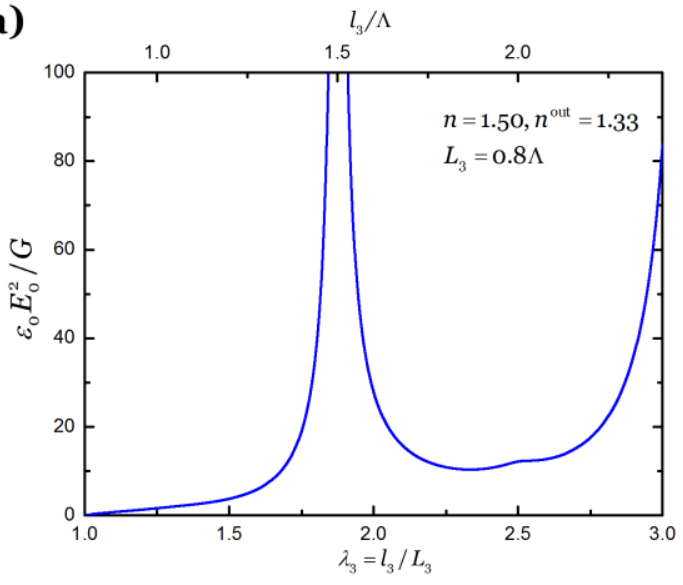

(c)

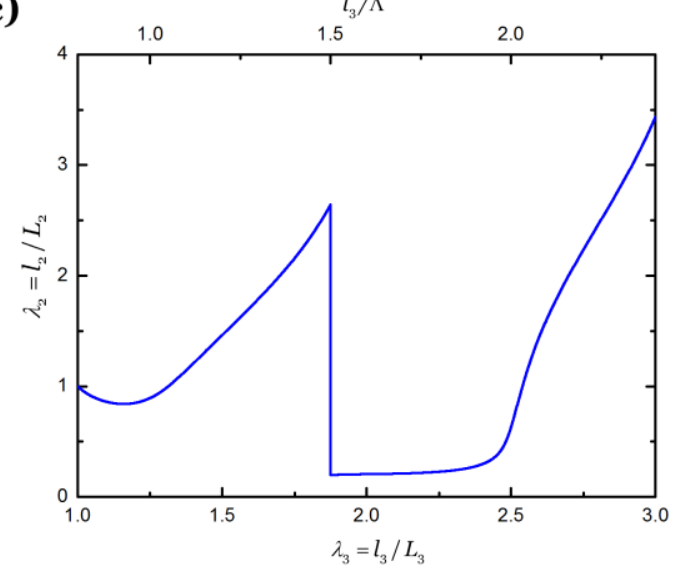

(b)

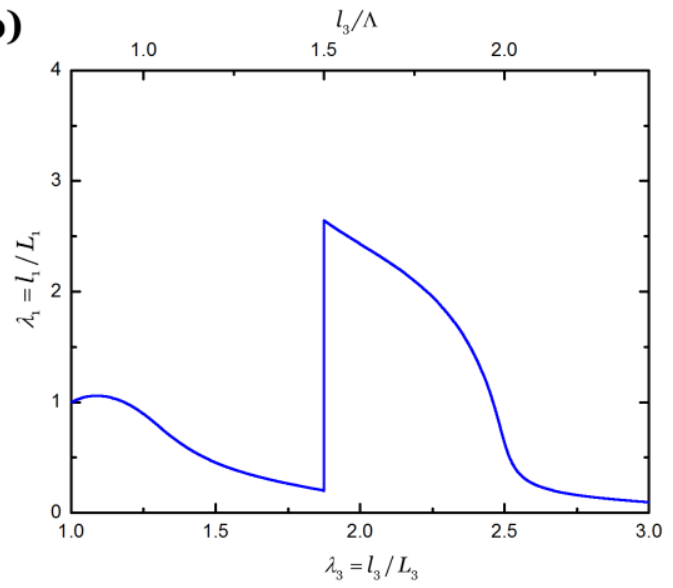

(d)

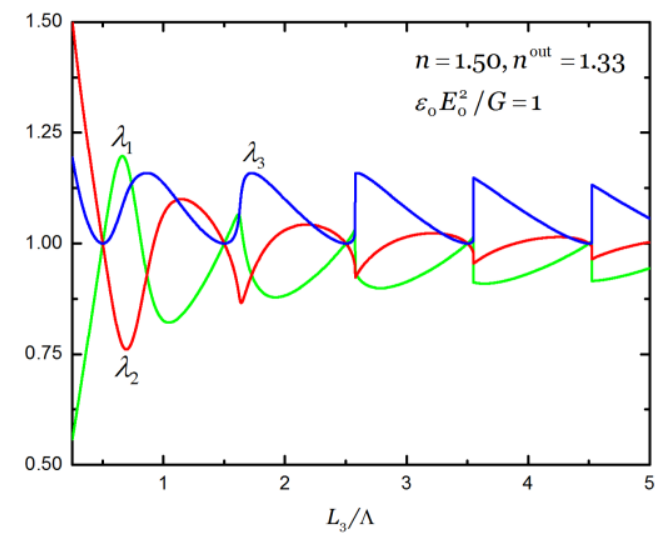

Fig. 7 Deformation induced by optical forces in a dielectric optically mismatched with the outside. (a) The out-of-plane stretch changes with the amplitude of the optical field. At $l_{3}=\Lambda$, the optical mismatch gives rise to a nonzero optical force, so that the deformation is maintained by a finite amplitude of the optical field, $E_{0}$. By contrast, at $l_{3}=1.5 \Lambda$, all components of the optical force vanish, so that the deformation cannot be maintained by an optical field of finite amplitude. (b) and (c) The in-plane stretches as functions of the out-of-plane stretch. (d) When the dielectric and the outside are optically mismatched, $\left\langle T_{3}^{\text {out }}\right\rangle \neq\left\langle T_{3}\right\rangle$ in general, and the out-ofplane component of the optical force deforms the dielectric even when $L_{3} / \Lambda \rightarrow \infty$. 
Table 1. Dimensionless numbers of optical forces relative to other forces

\begin{tabular}{|c|c|l|}
\hline types of motion & $\begin{array}{c}\text { dimensionless } \\
\text { numbers }\end{array}$ & meanings of parameters \\
\hline $\begin{array}{c}\text { Trap a rigid particle against } \\
\text { its Brownian motion. }\end{array}$ & $\frac{P}{c a^{2}} \frac{a^{3}}{k_{B} T}$ & $\begin{array}{l}k_{B} \text { : Boltzmann constant } \\
T: \text { temperature }\end{array}$ \\
\hline $\begin{array}{c}\text { Move a rigid particle in a } \\
\text { viscous liquid. }\end{array}$ & $\frac{P}{c a^{2}} \frac{a}{\eta v}$ & $\begin{array}{l}\eta: \text { viscosity } \\
v: \text { velocity of the moving } \\
\text { laser source }\end{array}$ \\
\hline $\begin{array}{c}\text { Deform an interface } \\
\text { between liquids against } \\
\text { surface tension. }\end{array}$ & $\frac{P}{c a^{2}} \frac{a}{\gamma}$ & $\gamma:$ surface energy \\
\hline $\begin{array}{c}\text { Bend a stiff material against } \\
\text { elasticity. }\end{array}$ & $\frac{P}{c a^{2}} \frac{1}{G}\left(\frac{L}{a}\right)^{4}$ & $G:$ shear modulus \\
\hline $\begin{array}{c}\text { Stretch a soft material } \\
\text { against elasticity. }\end{array}$ & $\frac{P}{c a^{2}} \frac{1}{G}$ & $G:$ shear modulus \\
\hline
\end{tabular}

